\title{
Intravenous Infusion of Nitroglycerine Leads to Increased Permeability on Dynamic Contrast-Enhanced MR Imaging in Pig Brains
}

\author{
J. Carl, D.A. Tideman, S. Ravn, K. Lund, S.O. Magnisdottir, and B. Kjærgaard
}

\begin{abstract}
BACKGROUND AND PURPOSE: It has been suggested that off-label use of transdermal nitroglycerine patches to prevent frostbite may lead to severe acute mountain sickness and ataxia. The aim of this study was to investigate the effect of nitroglycerine on brain vascular permeability by using dynamic contrast-enhanced MR imaging in a swine model.
\end{abstract}

MATERIALS AND METHODS: Eight Danish Landrace-Yorkshire-Danish Landrace pigs of approximately 20-25 kg were scanned with a dynamic contrast-enhanced MR perfusion protocol with and without nitroglycerine intravenous infusion. Compartmental analysis was performed on the basis of the Tofts model, and voxel-based quantitative values of the volume transfer constants from the vascular to the extracellular space were determined.

RESULTS: The scan with nitroglycerine infusion resulted in significantly higher volume transfer constant values than values derived from the first scan without nitroglycerine infusion. Increased volume transfer constant values were observed in 6 of 8 animals.

CONCLUSIONS: Infusion of nitroglycerine increases the vascular permeability of the swine brain on the basis of the transfer constant estimated from dynamic contrast-enhanced MR imaging.

ABBREVIATIONS: DCE-MR = dynamic contrast-enhanced MR imaging; ISP $=$ IntelliSpace Portal; $K^{\text {trans }}=$ volume transfer constant from the vascular to the extracellular space; MANOVA = multivariate analysis of variance; NTG = nitroglycerine

$\mathrm{N}_{\mathrm{t}}^{\mathrm{i}}$ itroglycerine (NTG) or nitrates are old drugs that were introduced into medical treatment $>100$ years ago for angina pectoris. The typical hemodynamic, anti-ischemic effects in humans were described in the 1950s and 1960s and are mediated through the release of nitric oxide in the walls of blood vessels, leading to relaxation of vascular smooth-muscle tone. ${ }^{1,2}$

Acute mountain sickness may affect mountaineers in high altitudes and has been ascribed to intracranial hypertension caused by extracellular vasogenic edematous brain swelling after mechanical disruption of the blood-brain barrier due to hypoxia. ${ }^{3}$ Off-label use of transdermal NTG patches to prevent frostbite may lead to severe acute mountain sickness and ataxia. ${ }^{3}$ Nitrateinduced cerebral vasodilation and high-altitude cerebral edema are theoretically possible on a pathophysiologic basis. Others have suggested that acute mountain sickness may be associated with

Received September 25, 2014; accepted after revision January 2, 2015.

From the Department of Medical Physics, Oncology (J.C., D.A.T., S.R., K.L.), and Biomedical Research Laboratory (S.O.M., B.K.), Aalborg University Hospital, Aalborg, Denmark.

Please address correspondence to Jesper Carl, PhD, Department of Medical Physics, Oncology, Aalborg University Hospital, Hobrovej 18-22, 9000 Aalborg, Denmark; e-mail: jhc@rn.dk

http://dx.doi.org/10.3174/ajnr.A4279 altered redox homeostasis and disordered cerebral autoregulation independent of barrier disruption. ${ }^{4,5}$ The cerebral hemodynamic effects of vasodilators are of clinical interest because a decrease in mean arterial pressure might alter global cerebral blood flow and brain perfusion. Increased permeability of the blood-brain barrier has been observed in NTG-treated animals (common carp, Cyprinus carpio $L$ ) on the basis of Evans blue capillary leakage into the brain tissue. $^{6}$

New imaging techniques to study brain perfusion and vascular permeability have emerged in the past few decades. One such technique is dynamic contrast-enhanced T1-weighted MR imaging (DCE-MR) by using diffusible agents such as gadopentetate dimeglumine. ${ }^{7}$ DCE-MR has the potential of characterizing abnormal capillary leakage. The use of pharmacokinetic compartment models to analyze DCE-MR data and obtain standardized quantitative parameters has been refined in recent years. ${ }^{8}$ These refinements have included the definition of the volume transfer constant $\left(K^{\text {trans }}\right)$ (minute $\left.{ }^{-1}\right)$ in the Tofts model, which represents the permeability surface area product per unit volume of tissue under permeability-limited or high-flow conditions. ${ }^{7}$ DCE-MR has been used in an MR imaging-integrated real-time swine intracerebral hemorrhage model to study acute hematoma growth and contrast extravasation. ${ }^{9}$ The purpose of the present study was 


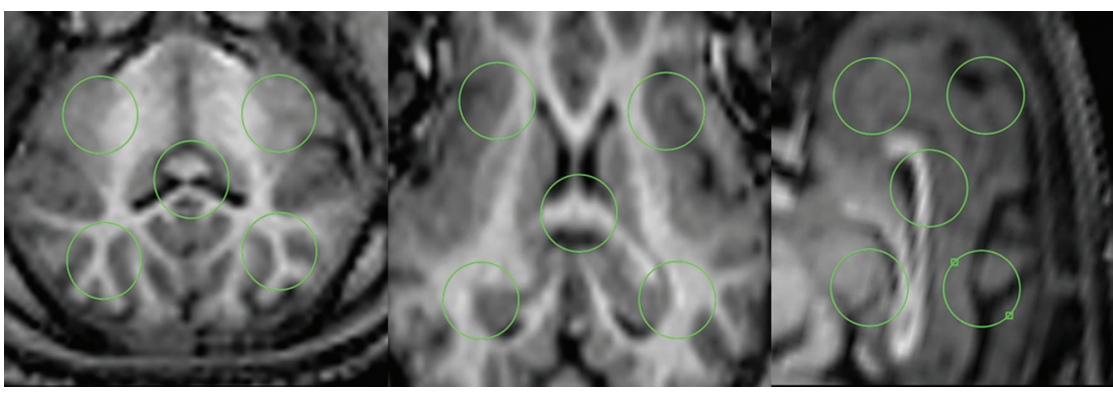

FIG 1. Example of the 5 ROIs (green circles) for reading mean $K^{\text {trans }}$ in each of the 3 different $\mathrm{Tl}$ turbo field echo scan planes of a pig brain. Panels from left to right are the axial, coronal, and sagittal planes.

to investigate the effect on brain permeability of administration of NTG by using DCE-MR in a swine model. The study was approved by the Danish Animal Experiments Inspectorate, reference number 2104-15-0201-00029.

\section{MATERIALS AND METHODS}

Eight Danish Landrace-Yorkshire-Danish Landrace pigs of approximately $20-25 \mathrm{~kg}$ were used in the present study. The animals were anesthetized by using tiletamine-zolazepam (Zoletil), a mixture of 2 dissociative anesthetics (ketamine, $6.25 \mathrm{mg} / \mathrm{mL}$, and tiletamine, $6.25 \mathrm{mg} / \mathrm{mL}$ ), a benzodiazepine (Zolazepam $6.25 \mathrm{mg}$ / $\mathrm{mL}$ ), a synthetic opioid (butorphanol, $1.25 \mathrm{mg} / \mathrm{mL}$ ), and xylazin $(6.5 \mathrm{mg} / \mathrm{mL})$; the mixture contains sedative, hypnotic, analgesic, and muscle-relaxing properties. This mixture was administered as an intramuscular injection at $1 \mathrm{~mL} / 10 \mathrm{~kg} / \mathrm{h}$ for a total of $2-2.5 \mathrm{~mL}$ every hour. After sedation was initiated, the animals were subsequently transferred to the scanner room. The animals were placed in a vacuum cushion on a table in a $3 \mathrm{~T} \mathrm{MR}$ imaging scanner (Ingenia 3T; Philips Healthcare, Best, the Netherlands) with the head and brain positioned within the standard head coil (15channel dStream HeadSpine coil; Philips). The animals were first scanned with a 3D T1 axial turbo field echo anatomy protocol with $\mathrm{TR}=8.1 \mathrm{~ms}, \mathrm{TE}=3.7 \mathrm{~ms}$, and voxel size $=0.9 \times 0.9 \times 1$ $\mathrm{mm}^{3}$. This scanning was used to obtain a high-resolution image of the anatomy in the pig's brain. Subsequently, the animals were scanned with a DCE-MR perfusion protocol. The DCE-MR protocol consisted of the following 3 scan sequences: a 3D T1 fastfield echo prescan sequence with flip angle $=6^{\circ}$, TR $=50 \mathrm{~ms}$, $\mathrm{TE}=1.7 \mathrm{~ms}$, and voxel size $=2.5 \times 2.5 \times 5.0 \mathrm{~mm}^{3}$. This sequence was followed by an identical prescan sequence, but with a flip angle of $16^{\circ}$. Finally, a dynamic 3D T1 turbo field echo sequence with a $\mathrm{TR}=4 \mathrm{~ms}, \mathrm{TE}=1.7 \mathrm{~ms}$; the same voxel size as in the prescans; and $12^{\circ}$ flip angles was used during the contrast infusion. All images had a 5-mm section thickness and 2.5- $\mathrm{mm}$ separation. Volume images were sampled at 4-second intervals for up to 7 minutes (434 seconds). For the dynamic acquisition, the MR imaging gadolinium contrast agent (gadoterate dimeglumine, Dotarem, 0.5 nm/mL; Guerbet, Aulnay-sous-Bois, France) was administered intravenously into an ear vein. With an automated injector, we administered a total of $2.5 \mathrm{~mL}$ of Dotarem at $3 \mathrm{~mL} / \mathrm{s}$ followed by a $20-\mathrm{mL}$ saline flush.

Compartment analysis was performed by using the MR perfusion tool in IntelliSpace Portal (ISP, Version 5; Philips), which uses the Tofts model. ${ }^{7}$ The ISP software uses the 2 flip angle pres- cans to estimate a true $\mathrm{T} 1$ relaxation map of the brain tissue. The ISP software then recalibrates the intensity in the DCE-MR dynamic series by using the T1 relaxation map, the Dotarem $r 1$ relaxation constant of 3.9 seconds $^{-1} \times$ $\mathrm{mmol}^{-1}$, and a hematocrit value of $45 \%$. After calibration, the MR signal is proportional to the concentration of the gadolinium concentration in the tissue. ISP then uses the recalibrated MR signal in a compartmental analysis based on the Tofts model. ${ }^{7}$ The standard arterial input function derived from a human population was used in the ISP software (population-averaged arterial input function) for the compartmental analysis with the Tofts model. The ISP analysis provided parametric volume images of the estimated tracer kinetic parameters in the Tofts model. This study particularly focused on the parametric images of $K^{\text {trans }}$ in the brain.

The animals were scanned on 2 different days, 2 weeks apart. On the first scanning day, the animals were subjected to 2 consecutive DCE-MR images, both without NTG, to evaluate the reproducibility of the method. On the second scanning day, the animals were subjected first to a DCE-MR image without NTG and, subsequently, a scan after NTG infusion. Before scanning, we loaded the injector with a $50-\mu \mathrm{g} / \mathrm{mL}$ NTG solution. The solution was injected at a rate of $0.07 \mathrm{~mL} / \mathrm{s}$ for 5 minutes. Infusion started immediately before the first prescan in the DCE-MR protocol. The total injected dose of NTG was $40 \mu \mathrm{g} / \mathrm{kg}$ during 5 minutes. After scanning, 15 circular ROIs were drawn on the image planes in the brain by 1 radiographer independent of the later statistical analysis. Each ROI had an area of $80 \mathrm{~mm}^{2}$ and was placed in a standardized and evenly distributed way in all 3 image planes, as shown in Fig 1. For each ROI, the mean value of $K^{\text {trans }}$ was calculated by using the ISP software. Statistical analysis was performed by using Statistica software (StatSoft, Tulsa, Oklahoma).

\section{RESULTS}

On the first scanning day, only 7 animals were scanned because scanning in 1 animal had to be abandoned due to respiratory distress symptoms when lying in the supine position. All 8 animals were scanned successfully on the second scanning day. Figure 2 demonstrates the effect of NTG infusion on the permeability in brain tissue. Increased permeability is observed as areas of confluent purple against the normal black background in the brain tissue when comparing the $K^{\text {trans }}$ parametric images with and without NTG infusion. The distribution of $K^{\text {trans }}$ values determined from the $K^{\text {trans }}$ maps was skewed; therefore, the numbers had to be logarithmically transformed to obtain a normal distribution of $K^{\text {trans }}$ values. Subsequently, multivariate analysis of variance (MANOVA) was applied by using $K^{\text {trans }}$ in the 2 subsequent DCE-MR images as dependent variables and animal ear tag, position, and image plane of ROI as categoric predictors. Valid MANOVA assumptions were tested by using a P-P plot to demonstrate normal distribution of $\log \left(K^{\text {trans }}\right)$ values qualitatively and the Levene test for variance homogeneity. Thus, mean $K^{\text {trans }}$ 


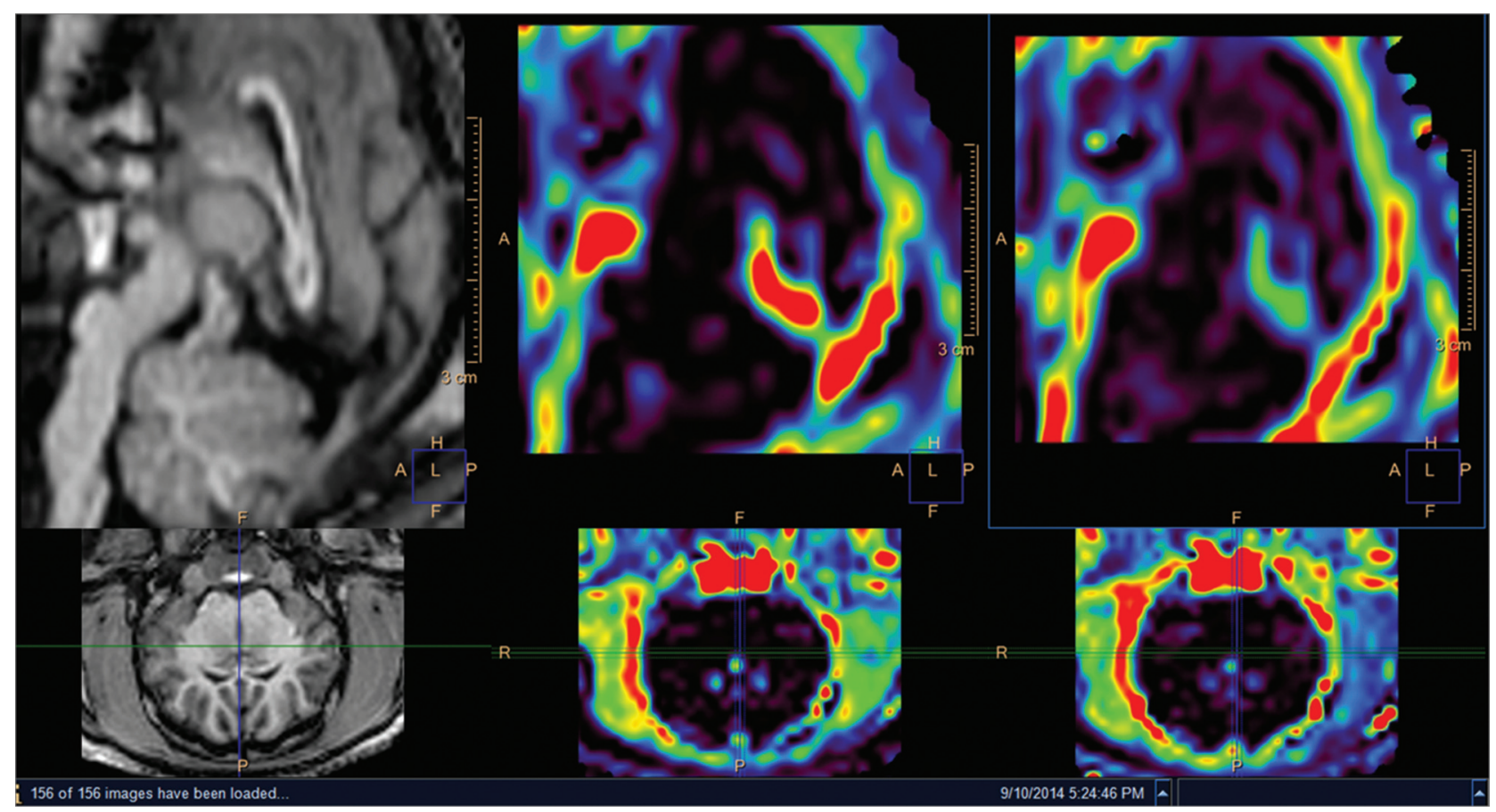

FIG 2. Left panel: axial and sagittal views of pig brain T1 turbo field echo scan. Middle panel: corresponding parametric $K^{\text {trans }}$ maps determined from a DCE-MR image of the same pig brain. Right panel: corresponding axial and sagittal views and similar parametric $K^{\text {trans }}$ maps determined from a DCE scan of the same pig brain after continuous infusion of nitroglycerine.

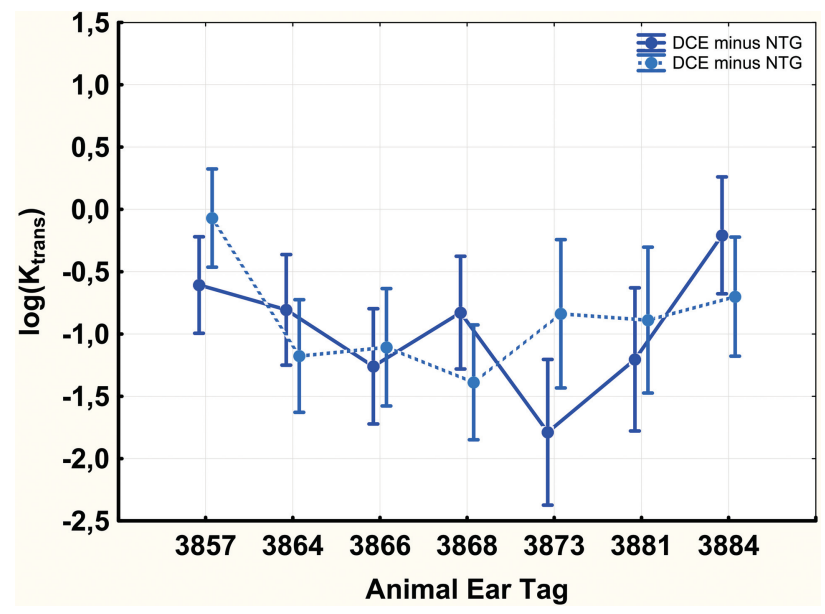

FIG 3. Plot of $\log \left(K^{\text {trans }}\right)$ from 7 different pigs (ear tag). The plot represents the mean values (filled circles) and the $95 \%$ confidence interval of the mean (error bars) for each animal. Means and SDs were determined by using a MANOVA analysis on 15 evenly distributed ROIs in the $K^{\text {trans }}$ volume map in the brain of each pig. The plot represents data from 2 different DCE-MR images obtained subsequently on the same day in each animal. $K^{\text {trans }}$ values determined in the 2 subsequent DCE-MR images, the first scan (dark blue solid line) and the second scan (light blue dotted line).

values in subsequent DCE-MR images in each animal could be corrected to influence the ROI from the position and image plane.

On the first scanning day, both DCE-MR images were obtained without infusion of NTG. The MANOVA analysis showed no significant changes in $K^{\text {trans }}$ values between the first and second (subsequent) DCE-MR images. Consequently, $K^{\text {trans }}$ values were reproducible for all 7 animals within the same scanning day, as demonstrated in Fig 3. On the second scanning day, the first DCE-MR image was obtained as on the first scanning day. Before

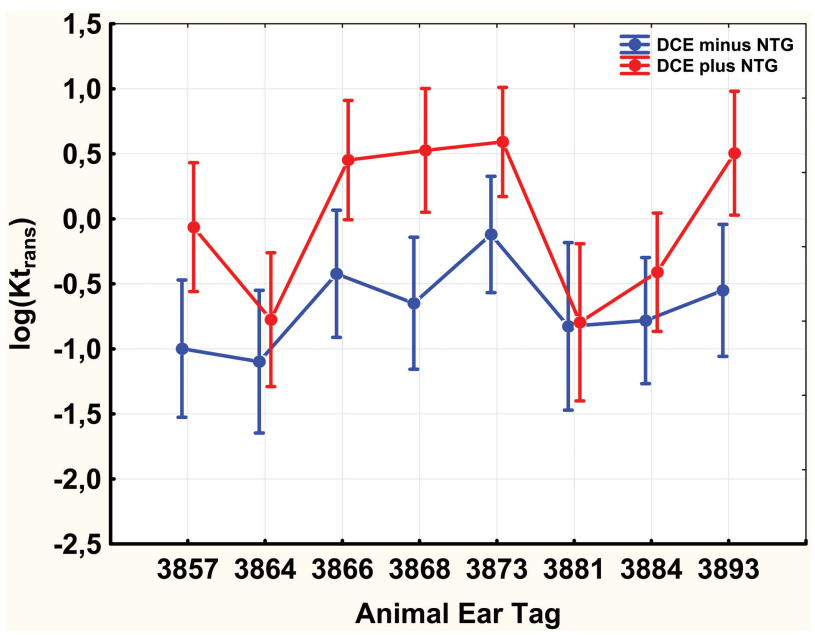

FIG 4. Plot of $\log \left(K^{\text {trans }}\right)$ from 8 different pigs (ear tag). The plot represents mean values (filled circles) and the $95 \%$ confidence interval of the mean (error bars) of each animal. Means and SDs were determined by using a MANOVA analysis of 15 evenly distributed ROIs in the $K^{\text {trans }}$ volume map in the brain of each pig. The plot represents data from 2 different DCE-MR images obtained subsequently on the same day in each animal. $K^{\text {trans }}$ values are from the first DCE-MR image (dark blue solid line) and the second DCE-MR image after infusion of nitroglycerine (red solid line).

the second DCE-MR image on the second scanning day, NTG was infused. Using the MANOVA analysis, we observed statistically significant higher estimated $K^{\text {trans }}$ values in 6 of the 8 animals after NTG infusion compared with values derived from the scan with no NTG infusion. The increase in $K^{\text {trans }}$ following NTG administration is shown in Fig 4. The corresponding values from the plot in Fig 4 are tabulated in Table 1. Analogously, similar results were obtained by using a paired $t$ test of log-transformed $K^{\text {trans }}$ values for each animal, as shown in Table 2. 
Table 1: Mean and standard error of the mean versus animal ear tag from the MANOVA analysis performed on $\log \left(K^{\text {trans }}\right)$ and plotted in Fig $4^{\text {a }}$

\begin{tabular}{lcccc}
\hline $\begin{array}{c}\text { Animal } \\
\text { Ear Tag }\end{array}$ & $\begin{array}{c}\text { Mean } \\
\text { (-NTG) }\end{array}$ & $\begin{array}{c}\text { SE } \\
\text { (-NTG) }\end{array}$ & $\begin{array}{c}\text { Mean } \\
\text { (+NTG) }\end{array}$ & $\begin{array}{c}\text { SE } \\
\text { (+NTG) }\end{array}$ \\
\hline 3857 & -1.00 & 0.26 & -0.06 & 0.25 \\
3864 & -1.10 & 0.28 & -0.78 & 0.26 \\
3866 & -0.42 & 0.25 & 0.45 & 0.23 \\
3868 & -0.65 & 0.25 & 0.53 & 0.24 \\
3873 & -0.12 & 0.22 & 0.59 & 0.21 \\
3881 & -0.83 & 0.32 & -0.80 & 0.30 \\
3884 & -0.78 & 0.24 & -0.41 & 0.23 \\
3893 & -0.55 & 0.25 & 0.50 & 0.24 \\
\hline
\end{tabular}

Note:-SE indicates standard error.

${ }^{a}$ The error bars in Fig 4 indicate the $95 \%$ confidence interval of the mean.

Table 2: The $P$ values in the last column show results the from paired $t$ test analysis on $\log \left(K^{\text {trans }}\right)$ in pig brains estimated from DCE-MR ${ }^{\mathrm{a}}$

\begin{tabular}{|c|c|c|c|}
\hline & -NTG & +NTG & Paired $t$ Test \\
\hline $\begin{array}{l}\text { Animal } \\
\text { Ear Tag }\end{array}$ & $\begin{array}{c}K^{\text {trans }}(95 \% \mathrm{Cl}) \\
\left(\mathrm{min}^{-1}\right)\end{array}$ & $\begin{array}{c}K^{\text {trans }}(95 \% \mathrm{Cl}) \\
\left(\mathrm{min}^{-1}\right)\end{array}$ & $\log \left(K^{\text {trans }}\right)$ \\
\hline 3857 & $0.4(0.2-0.9)$ & $1.0(0.4-2.3)$ & $P=.0003^{\mathrm{b}}$ \\
\hline 3864 & $0.4(0.2-0.9)$ & $0.5(0.2-1.3)$ & $P=.25$ \\
\hline 3866 & $0.7(0.3-1.8)$ & $1.7(0.9-3.1)$ & $P=.002^{b}$ \\
\hline 3868 & $0.6(0.2-1.5)$ & $1.8(1.1-2.8)$ & $P=.02^{b}$ \\
\hline 3873 & $0.8(0.4-1.7)$ & $1.7(0.9-3.0)$ & $P=.003^{b}$ \\
\hline 3881 & $0.7(0.3-1.7)$ & $0.7(0.2-1.7)$ & $P=.72$ \\
\hline 3884 & $0.5(0.2-1.1)$ & $0.6(0.3-1.4)$ & $P=.02^{b}$ \\
\hline 3893 & $0.6(0.3-1.3)$ & $1.8(1.0-3.3)$ & $P=.002^{b}$ \\
\hline
\end{tabular}

a The $t$ test demonstrated significantly higher values after intravenous infusion of nitroglycerine in 6 of 8 animals. The columns -NTG and +NTG represent backtransformed values with and without nitroglycerine, respectively.

b Significant.

\section{DISCUSSION}

This study demonstrated increased brain permeability estimated on DCE-MR after NTG was administered intravenously in a swine model. To our knowledge, this is the first time DCEMR has been used to visualize and measure the effects of NTG in the brain. The present study demonstrated a significant increase in brain permeability during the infusion of NTG in 6 of 8 animals. Only a relatively small number of studies exist on changes in brain functionality after the use of NTG in humans. The effects on cerebral hemodynamics from NTG administration as described in the literature are not fully understood and are sometimes ambiguous. Studies using MR angiography have demonstrated that NTG in humans increases the diameters of basal cerebral arteries. ${ }^{10,11}$ Intravenous infusion of NTG, $0.1-0.5 \mu \mathrm{g} /$ $\mathrm{kg}$, in healthy human volunteers has been studied by using $\mathrm{H}_{2}\left[{ }^{15} \mathrm{O}\right]-\mathrm{PET}$, and the study has shown that nitroglycerin increased global CBF. ${ }^{12}$ However, the use of transdermal NTG (5 $\mathrm{mg}$ ) in patients with acute stroke was not associated with changes in CBF, cerebral perfusion pressure, or cerebral steal when studied by using xenon-enhanced CT. ${ }^{13}$ Accordingly, others have demonstrated that the blood oxygen level-dependent MR signal was inversely related to $\mathrm{CBF}$ but did not change during administration of NTG in humans. This finding could indicate that NTG only exerts an effect on the large vessels. ${ }^{14}$ However, other studies have demonstrated contrary results. For example, the use of near-infrared spectrometry measuring cerebral oxygen saturation has demonstrated that NTG supports or may increase brain oxygenation. ${ }^{15,16}$ Analogously, another study demonstrated increased vascular diam- eter, blood flow, and endothelial permeability by using a different vasodilator, captopril (nitric oxide donator), in both in vitro and in vivo models. ${ }^{17}$ The results obtained in this study also indicate that the infusion of NTG has a significant effect on the vascular permeability in the brain of some but not all animals.

Large vessels in the brain may mimic increased permeability. When we placed the circular measuring areas in the 3 image planes in the brain, areas with large vessels were avoided. However, this was not entirely possible and may be why $K^{\text {trans }}$ values in some cases were relatively high, considering that an intact $\mathrm{BBB}$ is virtually impermeable to standard gadolinium-based contrast agents. This risk of including large vessels was the reason for using the MANOVA analysis with both animal reading plane and circle position as independent variables. Further studies are needed to understand this effect in detail. A better understanding of the mechanisms behind the effect of NTG on brain vasculature and hemodynamics is important because it might modulate the mechanisms of other drugs crossing the blood-brain barrier. ${ }^{18}$ Thus, it may implicate a therapeutic advantage in combining NTG with drugs that need to have a higher general availability in the brain.

\section{CONCLUSIONS}

Infusion of nitroglycerine increases the vascular permeability of the swine brain on the basis of the transfer constant estimated from dynamic contrast-enhanced MR imaging. Significantly increased permeability was observed in the brains of 6 of 8 Danish Landrace-Yorkshire-Danish Landrace pigs.

\section{REFERENCES}

1. Lichtlen PR. The mechanism of action of nitrates, 1988 status [in German]. Z Kardiol 1989;78(suppl 2):3-10; discussion 64-67

2. Dewey M, Hoffmann H, Hamm B. Multislice CT coronary angiography: effect of sublingual nitroglycerine on the diameter of coronary arteries. Rofo 2006;178:600-04

3. Mazzuero G, Mazzuero A, Pascariello A. Severe acute mountain sickness and suspect high altitude cerebral edema related to nitroglycerin use. High Alt Med Biol 2008;9:241-43

4. Bailey DM, Bärtsch P, Knauth M, et al. Emerging concepts in acute mountain sickness and high-altitude cerebral edema: from the molecular to the morphological. Cell Mol Life Sci 2009;66:3583-94

5. Cochand NJ, Wild M, Brugniaux JV, et al. Sea-level assessment of dynamic cerebral autoregulation predicts susceptibility to acute mountain sickness at high altitude. Stroke 2011;42:3628-30

6. Kovacic S, Petrinec Z, Matasin Z, et al. Increased permeability of the blood-brain barrier following administration of glyceryl trinitrate in common carp (Cyprinus carpio L.). Coll Antropol 2008;32(suppl 1):99-103

7. Tofts PS, Brix G, Buckley DL, et al. Estimating kinetic parameters from dynamic contrast-enhanced T(1)-weighted MRI of a diffusable tracer: standardized quantities and symbols. J Magn Reson Imaging 1999;10:223-32

8. Tofts PS. Optimal detection of blood-brain barrier defects with GdDTPA MR: the influences of delayed imaging and optimised repetition time. Magn Reson Imaging 1996;14:373-80

9. Aviv RI, Huynh T, Huang Y, et al. An in vivo, MRI-integrated realtime model of active contrast extravasation in acute intracerebral hemorrhage. AJNR Am J Neuroradiol 2014;35:1693-99

10. Niehaus L, Gottschalk S, Weber U. Effect of drug-induced vasodilatation of basal brain arteries with nitroglycerin on blood flow velocity and volume flow in the middle cerebral artery [in German]. Ultraschall Med 1998;19:225-29

11. Siepmann M, Kirch W. Effects of nitroglycerine on cerebral blood 
flow velocity, quantitative electroencephalogram and cognitive performance. Eur J Clin Invest 2000;30:832-37

12. Bednarczyk EM, Wack DS, Kassab MY, et al. Brain blood flow in the nitroglycerin (GTN) model of migraine: measurement using positron emission tomography and transcranial Doppler. Cephalalgia 2002;22:749-57

13. Willmot M, Ghadami A, Whysall B, et al. Transdermal glyceryl trinitrate lowers blood pressure and maintains cerebral blood flow in recent stroke. Hypertension 2006;47:1209-15

14. Asghar MS, Hansen AE, Pedersen S, et al. Pharmacological modulation of the BOLD response: a study of acetazolamide and glyceryl trinitrate in humans. J Magn Reson Imaging 2011;34:921-27
15. Kadoi Y, Saito S, Morita T, et al. The differential effects of prostaglandin $\mathrm{E} 1$ and nitroglycerin on regional cerebral oxygenation in anesthetized patients. Anesth Analg 1997;85: 1054-59

16. Hare GM. Nitroglycerin and cerebral oxygen saturation. Can J Anaesth 2008;55:52-53; author reply 53

17. Jia $\mathrm{L}$, Wong $\mathrm{H}$. In vitro and in vivo assessment of cellular permeability and pharmacodynamics of S-nitrosylated captopril, a nitric oxide donor. Br J Pharmacol 2001;134:1697-704

18. Abe H. Effects of combination of vasodilator drugs and hypertonic solutions on methotrexate distribution into the rat brain. Jpn J Pharmacol 1988;48:373-76 